\title{
La place de la métaphore en sociologie
}

\section{Giovanni Busino}

\section{OpenEdition \\ Journals}

Édition électronique

URL : http://journals.openedition.org/ress/539

DOI : $10.4000 /$ ress.539

ISSN : 1663-4446

\section{Éditeur}

Librairie Droz

\section{Édition imprimée}

Date de publication : 1 septembre 2003

Pagination : 91-101

ISBN : 2-600-00878-0

ISSN : 0048-8046

Référence électronique

Giovanni Busino, "La place de la métaphore en sociologie ", Revue européenne des sciences sociales [En ligne], XLI-126 | 2003, mis en ligne le 30 novembre 2009, consulté le 30 avril 2019. URL : http:// journals.openedition.org/ress/539; DOI : 10.4000/ress.539 


\section{Giovanni BUSINO}

\section{LA PLACE DE LA MÉTAPHORE EN SOCIOLOGIE}

Dans toutes les activités de modélisation, les modes de pensée métaphoriques, c'est-à-dire le transfert de sens d'un domaine à l'autre, le générateur de formes rendant visible ce qu'on ne voit pas, la réduction de l'étranger au familier, la chaîne des liens entre les différents objets, ont un rôle heuristique important bien que lourd de nombreux paralogismes. Dans les sciences sociales, dans ces disciplines qui ne peuvent ni ne savent construire leurs objets comme des objets formels, qui forment leurs concepts par différences ou similitudes, contrastes ou dégradés entre les éléments d'une série d'observations et dont le raisonnement est foncièrement comparatif, ce rôle est déterminant, il est presque un principe d'économie, un rasoir d'Occam.

Les schèmes métaphoriques tiennent ensemble les rapports hétérogènes, les isotopies, ils favorisent l'extension du sens antérieur d'un signifiant et I'attribution de deux sens un peu différents au même mot (l'antanaclase), ainsi que I'intégration de perspectives variées, même si les variables construites ne sont pas comparables entre elles sous tous les rapports. Sans ces schèmes l'élaboration des présupposés, des catégories, des grilles de description, des protocoles de vérification ne serait pas possible et le chercheur ne pourrait plus «fabriquer» du sens, donner des formes au réel, argumenter sur des objets dont les significations restent indexées sur des référents singuliers susceptibles d'interminables rectifications sémantiques. Le chercheur ne saurait plus comment s'accommoder de l'incapacité des systèmes sociaux à s'autosuffire sémantiquement.

En somme, grâce à ces schèmes métaphoriques nous pouvons analyser les processus sociaux, agencer les représentations du social, généraliser les descriptions des phénomènes échappant aux transcriptions directes, aller au-delà des énumérations des caractères des phénomènes observables, arriver à circonscrire des nouveaux champs perceptifs, lier la conceptualisation de la description à celle de l'explication, réorganiser l'instabilité des données et des énoncés, multiplier les constats plus ou moins pertinents.

Bien que l'importance de ces schèmes pour la connaissance sociologique soit avérée, nous sommes toujours dépourvus d'une théorie propre à distinguer les bonnes des mauvaises métaphores, les modèles (purs à la Hotelling ou déictiques à la Hapkins) des métaphores, les analogies de proportion de celles d'attribution.

Est-ce que l'histoire des usages et des modi operandi des métaphores, des synecdoques, des métonymies et des autres tropes et paralogismes à l'œuvre dans les sciences sociales pourraient nous aider à pallier cette carence? Est-ce que l'histoire de ces pratiques pourrait nous aider à comprendre pourquoi les analyses sociologiques fondées sur les métaphores, analogiques ou iconiques, enferment, 
en toutes circonstances, des contenus intuitifs, restent les produits d'intuitions, de rapprochements, d'emprunts à des contextes présumés similaires?

L'histoire montre qu'en sociologie la métaphore délocalise à la fois «un mot vers un sens différent de son sens originel », donne «à un objet un nom qui désigne un autre objet», mais également elle transfère les systèmes conceptuels, soutient les échanges d'un niveau ou degré à d'autres niveaux ou degrés. Même en l'absence de critères de proportion et de correspondance, même quand les «objets » n'existent que grâce aux conceptualisations et aux théories les ayant construits, même lorsque la métaphore privilégie un élément au détriment de tous les autres et dissimule les différences ou les ramène à l'identité, le chercheur arrive à mettre en place des approches analytiques stéréoscopiques dotées de sens. Grâce à la métaphore le chercheur forme les nouveaux prédicats d'observation, regarde les phénomènes sociaux «comme si», schématise les relations entre des énoncés et les rend intelligibles en tant qu'objets particuliers d'expérience. Mais coordonner des énonces où la schématisation joue un rôle essentiel implique forcément le recours à des chaînes de métaphores.

L'organicisme offre, à ce propos, des exemples significatifs. Les métaphores biologiques pour l'interprétation des phénomènes sociaux ont été utilisées dès l'aube de la sociologie. Pour les rendre intelligibles, la sociologie a fait appel aux figures de l'organisme biologique et puis elle les a détaillées et ajustées avec les métaphores de la sélection, de l'hérédité, de la lutte pour la vie, des facteurs biosociaux et démographiques. La société a été ainsi reconstruite en termes de complexité, de différenciation, de compartimentage systémique. La sociobiologie de Edward O. Wilson, pour rendre intelligibles les comportements humains, fait des larges usages de métaphores empruntées à l'évolution, à l'éthologie, à la génétique des populations, à l'herméneutique des comportements des animaux sociaux.

Une étude exhaustive de ces usages, depuis l'époque de Comte et Spencer jusqu'aux fonctionnalistes du $\mathrm{XX}^{\mathrm{e}}$ siècle, en cours de réalisation, permettra, nous l'espérons, d'évaluer non seulement les modes d'utilisation des métaphores dans la construction des objets d'expérience, des représentations sociales, mais aussi de formaliser des pratiques sociologiques courantes. Cette étude aidera, peut-être, à expliquer comment nous manipulons les similarités, les identités, les différences dans les argumentations en langue naturelle, comment nous arrivons à intégrer, en des ensembles hétéroclites, des multiples perspectives et à rendre recevables les énoncés ainsi construits.

Les propriétés stéréoscopiques des métaphores ont une forte puissance explicative et pourtant nous savons que celles nommées par les logiciens «métaphores iconiques » possèdent une toute petite partie des propriétés du signe visuel représentatif. Ces métaphores font référence à elles-mêmes au sein d'un même système, parfois aux jeux des interrelations entre une catégorie quelconque et l'un ou l'autre des spécimens pris en considération. Certes, elles aident à décrire les phénomènes, mais sans comparaison des ressemblances et/ou des différences.

Vilfredo Pareto nous en donne un exemple lorsqu'il utilise la métaphore de la toupie renversée ou de la flèche pointue ou de la pyramide pour étayer sa théorie de la distribution de la richesse dans les sociétés du passé et du présent, ou encore quand il fait appel à la géologie pour théoriser la circulation verticale, ascendante et descendante, des élites entre les différentes couches et strates de la société. Un 
autre exemple, plus connu, est donné par Lévi-Strauss avec sa métaphore du bricolage appliquée aux faits culturels et puis devenue la charpente de sa théorie de la pensée mythique.

D'une autre nature sont les «métaphores analogiques», impliquant un système de relations d'égalité entre les espèces et les catégories. Ces «métaphores analogiques » créent des images par comparaison ou par contraste et laissent entrevoir à quoi ces images ressemblent. L'épidémiologie, par exemple, a permis d'analyser la propagation, la fréquence, la distribution et l'évolution des rumeurs sociales tandis que l'oncologie d'élaborer la doctrine du racisme en tant que cancer moulinant le tissu social. Le raisonnement par induction, qui passe, à la suite d'inférences successives, du particulier au général, nous offre un autre exemple.

C'est au moyen des «métaphores analogiques» permettant l'assomption de certaines similitudes entre différents rapports, qu'on a pu formuler différentes théories de portée moyenne et certaines représentations du réel.

Les «métaphores illustratives» permettent, quant à elles, de faire appel à un simili et à un comme si. Elles dispensent de la comparaison à dimensions multiples et métamorphosent ce qu'elles sont supposées illustrer. Gordon Tullock utilise ce genre de métaphores illustratives lorsqu'il applique à la politique et à la bureaucratie la théorie du marché (monopole, concurrence, coût d'information, etc.), à l'électeur la théorie du consommateur et lorsqu'il convertit la rationalité économique en rationalité politique et la somme des intérêts individuels en intérêts collectifs.

Les métaphores qui prétendent décrire des univers déterminés ou des contenus constituent, pour faire court, une sorte d'ontologie implicite de la sociologie. Les plus courantes sont: (1) Le mécanicisme (traiter les phénomènes sociaux comme des variétés des phénomènes physiques); (2) L’organicisme (rendre intelligibles les phénomènes sociaux en s'appuyant sur les sciences de l'organisation du vivant); (3) L'économisme (concevoir l'acteur social comme capable d'intentions, de stratégies, de calcul, de rationalité instrumentale et procédurale); (4) Le sociologisme (prééminence de la totalité sur les parties la constituant, la société surplombe les individus); (5) Le langage (l'action réciproque des êtres humains et les signes qui la rendent visible ne sont possible que par l'appartenance à une communauté de significations d'où la primauté des signes langagiers); (6) La mise en scène théâtrale, la dramaturgie (les comportements sont régis par le cadre définissant la situation, d'où les emprunts aux rituels de présentation et d'interaction du théâtre); (7) Le jeu (dans les situations d'interaction de caractère stratégique l'agent est un joueur qui vise à maximiser ses avantages); (8) Le réseau (tantôt cette métaphore facilite le refus de la dichotomie nature/société, science/politique, sujet/objet, homme/animal, etc., la totalité devient une structure contraignante, constituée d'éléments liés par des degrés d'interdépendance et faisant émerger des relations et des interactions, tantôt elle simplifie l'adhésion au paradigme des technologies de l'information et offre les bases matérielles pour son extension à la structure sociale tout entière).

L'utilisation de ces huit métaphores de base (mais il y en a d'autres moins pratiquées) varie d'un sociologue à l'autre, elle n'est ni constante ni uniforme.

Prenons le cas de la métaphore tirée de (1) la mécanique ou de la thermodynamique. Pareto y fait constamment référence. La théorie de l'équilibre est au centre 
de toutes ses analyses. La métaphore de l'équilibre (construite sur l'idée d'égalité de force entre deux ou plusieurs réalités opposées et sur l'idée de repos découlant pour ceux qui s'y trouvent soumis) ainsi que les notions de stabilité, instabilité, réelles et virtuelles, d'équilibre dynamique, lui servent pour tenir ensemble et puis analyser les aspects de phénomènes économiques et sociologiques. Talcott Parsons, lui aussi, fonde sa théorie du système social sur la notion d'équilibre. Mais ni Pareto ni Parsons ne se rallient à la thèse selon laquelle l'équilibre du système est garanti au moment où tous ses composants sont en position d'immobilité et ils y reviennent spontanément si une perturbation provient de l'extérieur. Les deux donnent à la notion des significations si différentes (entropie maximale, introduction d'un ordre diminuant l'entropie, processus autonome d'équilibration, équilibre des conditions permanentes, activités et ouvertures dans les échanges, processus fonctionnel différent de la structure, etc.) que chacune d'elles peut être conçue comme une métaphore en soi, par ailleurs difficilement comparable ou intégrable avec les autres dans un ensemble unitaire. Et pourtant chacune d'elles, généralisant certaines conclusions à un autre domaine, à un autre niveau d'action, éclairent l'objet étudié, corrèlent les imbrications des contextes et des relations, organisent les phénomènes perçus par les sens et les rendent intelligibles en première approximation.

Les métaphores tirées de (2) L'organicisme sont encore plus nombreuses. Chez les pères fondateurs de la sociologie, elles se marient avec les mécanicismes et n'ont que des liens assez lâches avec la pratique méthodique de traitement des informations empiriques. On peut même ajouter que les métaphores organiques constituent le lieu commun le plus ancien et le plus utilisé des représentations de la société. D'elles, par des procédures inférentielles hasardeuses et des hypothèses risquées, par des opérations indifférentes aux règles des démarches empiriques, Spencer et Durkheim ont dérivé la théorie du passage du simple au complexe; Comte, Marx, Tylor, Morgan celles des étapes ou des stades du développement; Parsons et Merton la théorie de l'évolution sociale et Smelser la théorie du comportement collectif et celles du progrès et du changement.

L'organicisme a permis de concevoir la société comme un organisme biologique, en tant que système relativement fermé, ayant une finalité prépondérante, celle de survivre et de croître. Pour réaliser cette finalité, la société doit s'adapter au milieu et mettre en place des stratégies complexes afin de se produire et de se reproduire. Les divers types de sociétés (société de chasse et de cueillette, pastorale, horticole, agricole, industrielle, etc.) correspondent aux stades de développement de l'organisme vivant. Les changements sont engendrés par les lois naturelles du développement. Ils relèvent rarement du hasard; ils sont lents, continus, progressifs, linéaires, nécessaires, endogènes. Les sociétés primitives sont celles dont l'évolution ne fait que commencer ou qui s'est bloquée. D'où les métaphores analogiques, utilisées par Jean Piaget, entre les enfants et les primitifs, entre les stades et périodes dans le développement de l'intelligence chez l'enfant et l'histoire des sciences, entre la psychologie génétique et l'épistémologie. Les analogies biologiques permettent d'identifier dans les sociétés primitives les facteurs de croissance des sociétés modernes, mais à une phase antérieure de leur évolution. Elles deviennent ainsi les présupposés de la plupart des théories du changement, du développement et de la modernisation.

Que la société soit un organisme dont les fonctions, par ailleurs indéfinissables en termes de conduites observables empiriquement, visent de façon téléologique 
à en garantir la survie, ce postulat d'universalité évolutive, transposé mécaniquement de l'organisme biologique à l'organisme social, demeure, pour le moment, très problématique: sa logique est circulaire, ses hypothèses invérifiables, ses généralisations irréfutables, ses apories nombreuses et ses contradictions internes caractérisées par d'insurmontables difficultés conceptuelles. Il n'en reste pas moins que les différentes versions de cette métaphore organique aident à circonscrire certains phénomènes autrement indiscernables, à réduire la complexité, et, pour ces raisons, elles continuent à prospérer, ainsi que nous le prouvent les travaux d'Edward 0. Wilson et la sociologie de l'évolution bio-culturelle de Joseph Lopreato.

Les sociologues font aussi appel aux métaphores économiques (3), notamment à cause de la proximité entre les sciences naturelles et l'économie. Certains d'entre eux, fascinés par les progrès de cette dernière discipline, pensent que l'adoption de la méthodologie économique sortira la sociologie de l'impasse. Dès lors, ils empruntent à l'économie néoclassique son postulat fondateur: la société se compose d'individus juxtaposés, recherchant leur intérêt de manière rationnelle. Ils entrent en contact les uns avec les autres par l'intermédiaire du marché afin de maximiser leurs intérêts. Un ordre est ainsi créé grâce aux mécanismes d'ajustement automatique du marché. Les agents cherchent toujours à atteindre leur propre position d'équilibre. Chaque agent est doté de volonté-liberté et de capacité-efficacité, qualités indispensables pour réaliser des objectifs. Les sociologues vont appliquer la métaphore du marché aux sphères non marchandes, voire à tous les aspects de la vie sociale. Ils pensent, à la suite de Gary S. Becker, que «la théorie économique est peut-être en passe de fournir un cadre unifié à tout comportement qui met en œuvre des ressources rares, non marchandes, non monétaires aussi bien que monétaires, internes à un groupe restreint aussi bien que concurrentiel.» Ils sont même convaincus que: «Tout comportement humain peut être conçu comme mettant en jeu des participants qui maximisent leur utilité à partir d'un ensemble stable de préférences et qui accumulent la quantité optimale d'informations et autres intrants sur une variété de marchés.»

Cette métaphore économiciste implique, entre autres, la constitution de toutes les sciences sociales en économie généralisée des comportements humains, du mariage à la criminalité, de l'adultère à la justice, des associations sans buts lucratifs au marché religieux, du vote électoral à l'assistance aux pays en voie de développement. Chaque individu ne trouve le sens de ses actes qu'à maximiser ses utilités. Produire ces mêmes utilités de façon efficace reste le seul, l'ultime enjeu de ses choix qui portent sur le temps, le seul bien vraiment rare. Un exemple tiré de A Theory of Marriage, servira à illustrer ces propos. Dans ce livre publié en 1981 Becker utilise métaphoriquement la théorie de l'entreprise pour analyser la famille, car la famille est comparable, dit-il, à une «petite usine». Le contrat de mariage est le résultat d'un processus de tâtonnement sur le marché du mariage. L'enjeu - le choix du conjoint - concrétise la maximisation des utilités liées de deux individus. Les mariés produisent des satisfactions finales, objets d'une fonction d'utilité familiale qui contribue à transformer toute consommation en production. La formalisation de ces utilités est possible, le temps étant un bien rare dans la fonction de production domestique.

Mais dans une situation de maximisation des utilités sous contraintes (contrainte budgétaire, contrainte temporelle, ou autres), comment les membres 
d'une famille vont-ils agir? En utilisant leur temps entre la production de revenus par le travail salarié et la satisfaction de certains besoins d'origine non marchande.

De tels emprunts permettent, certes, de traiter la famille comme s'il s'agissait d'une entreprise, cependant ils nous forcent à considérer le temps dans la famille comme l'équivalent du temps du travail salarié, à confondre le privé avec le public, à escamoter le fait que dans le travail domestique, il y a une part non mesurable, car invisible socialement, à ignorer que les échanges ne peuvent pas tous être transcrits en langage monétaire et formalisé ainsi. Derrière tout cela, il y a, bien entendu, une représentation métaphorique de la société en tant que mécanisme de régulation automatique, en tant qu'ordre naturel, en tant que système physique ou organique.

Ces emprunts métaphoriques ont donné naissance à un important courant intellectuel que l'on nomme aujourd'hui «théorie de l'action rationnelle», «Rational Action ou RAT ou encore Rational Choice». Il regroupe des tendances différentes: la «logique de l'action collective», le «rationalisme méthodologique », «actionnalisme» et l'«individualisme méthodologique». Tous les tenants de ce courant empruntent à l'économie néoclassique la métaphore des acteurs sociaux agissant en fonction de leur préférence, et donc se comportant de manière rationnelle. La tendance purement économiciste exclut, en principe, de sa rationalité la solidarité, le pouvoir et le prestige; celle du rationalisme les comportements gouvernés par des normes et des règles; celle de l'actionnalisme les croyances et les préférences; celle de l'individualisme méthodologique n'importe quelle forme de totalité. Tous ces courants n'aboutissent point aux mêmes résultats. Par exemple, I'individualisme méthodologique peut renverser ou éliminer son économisme de départ, alors que le rationalisme économique doit rompre avec l'individualisme méthodologique pour rester fidèle à ses corollaires (agir de façon égoïste, exclusivement pour ses intérêts, se contenter de «satisficying" plutôt que de maximisation ou d'optimisation alors même que divers facteurs affectent la fonction d'utilité, que I'information n'est pas toujours correcte et l'environnement de l'action n'est pas toujours constant et donné).

La métaphore sociologiste (4) a en Durkheim son maître-d'œuvre. L'auteur de Les règles de la méthode sociologique, ainsi que François Bourricaud l'a montré de façon lumineuse en 1975, n'est jamais arrivé à s'arracher aux enchantements et aux facilités des approches mécaniste et organiciste, ni à se débarrasser de la croyance qu'il n'y a d'autres voies dans l'étude des sociétés que celles qui sont fournies par les règles, les procédures et les raisonnements déductifs et inductifs, par les modèles de rationalité des sciences physiques. Durkheim répète que les faits sociaux sont des choses, qu'ils n'ont rien à faire avec la psychologie, qu'ils sont des représentations d'un sens collectif, que la société est un super-organisme précédant et constituant les individus ainsi que leurs sentiments et leurs croyances. La conscience collective ne dépend pas ontologiquement des intentionnalités individuelles, elle n'est pas la résultante des comportements individuels à expliquer. Elle est génitrice d'histoire mais entièrement étrangère à l'histoire. Ces flux d'emprunts conceptuels, ces usages métaphoriques de concepts élaborés à l'abri des astreintes spatio-temporelles, font que l'explication sociologiste se base sur des concepts réifiés, qu'elle donne lieu à un savoir aux frontières incertaines, à l'identité nébuleuse, à des résultats cognitifs contestables, à des nombreuses contradictions internes, mais aussi à des incohérences de 
méthodes, comme celles que les catégories de l'anomie et du fatalisme produisent dans le cadre de l'analyse causale.

La culture et la langue (5), en tant que charpente de la société, en tant que système d'expression et de communication pour définir l'action sociale, ont commencé, très tôt, à intriguer les sociologues. Marcel Mauss a souvent écrit que le social constitue un texte à lire, que les comportements doivent être déchiffrés en tant que rapports d'échanges, de réciprocité et d'équivalence, que les faits sociaux sont des réalités symboliques, dotées de règles grammaticales, avec des morphèmes et des phonèmes. Depuis, pour enraciner la réalité sociale dans l'univers symbolique, trois approches ont été élaborées par les sociologues: l'interactionnisme symbolique, l'ethnométhodologie et le structuralisme. En appliquant métaphoriquement à la sociologie plusieurs schémas analytiques de la linguistique générale, et notamment ceux de diachronie/synchronie, forme/substance, langue/parole, logique/langage, sens/signe, les sociologues ont cru mettre en évidence les systèmes de règles qui régissent la société, son fonctionnement ici et maintenant, ils ont cru découvrir le mode de production des significations ainsi que la modalité de construction de la réalité sociale. Néanmoins, ils n'ont jamais pu caractériser la spécificité de la langue par opposition aux constructions sociales qu'elle serait capable d'élaborer et de façonner. Si la langue organise et conditionne notre manière de concevoir le monde, nos comportements sociaux, nos systèmes symboliques, doit-on alors dire que l'ordre social est langue? Mais puisque ni la langue ni la société n'ont un statut ontologique transcendantal, puisque ni la linguistique générale ni la sociologie ne possèdent d'unités concrètes susceptibles d'être immédiatement reconnues, les sociologues se sont trouvés dans une impasse. Cependant, les emprunts linguistiques transformés en métaphores et en canons heuristiques ont permis à Herbert Blumer et à Erwing Goffman, à Harold Garfinkel et à Aaron Cicourel de montrer comment se mettent en place les opérations de désignation moyennant lesquelles les objets sociaux et le système social sont construits. L'analyse conversationnelle de Harvey Sacks et de E. A. Schegloff, les logiques argumentatives de Stephen Toulmin et de JeanBlaise Grize ont ouvert de belles perspectives sur la persuasion et les logiques non démonstratives de la communication et en général sur les représentations connotatives du monde quotidien. De la métaphore linguistique la sociologie a tiré deux hypothèses majeures: (a) Le langage parlé est un système de signes parmi d'autres; (b) Le système de signe a un caractère hermétique et autoréférentiel.

L'usage que Claude Lévi-Strauss a fait de ces deux hypothèses pour analyser les règles du mariage et de la parenté, ainsi que les mythologiques, les symboles et leurs doubles, est bien connu. L'analyse des systèmes de signes verbaux et non verbaux a obligé les sociologues à établir des liens entre la conscience historique et les aspects normatifs de la science, à s'intéresser à l'herméneutique, à l'interprétation, à la lecture des systèmes culturels, à décoder les comportements individuels et sociaux gouvernés par des normes et des règles, les actions élaborées en fonction de modèles normatifs et de systèmes de règles et gouvernés par la cohérence, la pertinence et la complétude qui caractérisent tout modèle normatif et tout système de règles.

Cependant la métaphore linguistique, indifférente soit à la connaissance absolue soit à celle exprimée en termes de probabilité statistique, pose des problèmes compliqués et difficiles à résoudre. Si l'objet est construit grâce au 
processus de désignation, si la langue définit la situation et la montre, il est alors impossible de caractériser les messages, les informations, les sensations. Les émotions; il est problématique de réduire la désignation de l'objet à sa représentation. Si la définition et la compréhension de la situation découlent du processus de désignation qui ne distingue pas les relations sociales en tant que langage et le langage en tant que relations sociales, la connaissance reste fatalement limitée à un univers particulier et spécifique et dont la clôture sémantique est inéluctable.

La métaphore théâtrale, la mise en scène, la dramaturgie (6) présument que le monde social serait une scène et que chaque individu y tient un rôle. Or il n'y a aucun isomorphisme entre la scène théâtrale et la vie sociale, entre les sujets sociaux et les acteurs, entre l'intrigue d'une pièce de théâtre dont le déroulement et la fin ont été agencés préalablement et qui sont donc connus à l'avance, et l'imprévisibilité de l'enroulement de l'existence sociale faite souvent d'actions aux conséquences non voulues et de réactions et résultats parfois aux effets paradoxaux ou pervers. Les expériences théâtrales sont momentanées et passagères, imaginées et ordonnées par un auteur, prédéterminées et préstructurées par lui seul; elles vivent durant l'espace d'une représentation, d'un spectacle, elles sont prévisibles et toujours à distance du spectateur, alors que les expériences sociales se font au hasard des improvisations de tout un chacun, elles sont duratives, inévitables, nous entourent de tous les côtés et leurs conséquences restent imprévisibles, non préméditées, non volontaires, non structurées. Pour cela la vie sociale ne peut même pas être comparée à une «commedia dell'arte», puisque les acteurs ici nouent et dénouent une trame donnée à l'avance, qu'ils peuvent inventer des reparties inopinées, alors que, dans la première, les aléas et les contraintes normatives déterminent la portée des actions, la liberté limitée de l'agent social. Toutefois la métaphore dramaturgique apporte quelques lumières lorsqu'elle est appliquée à certains domaines spécifiques de la vie sociale (cérémonies initiatiques, rites de passages, conflits de rôles, crises, etc.) ainsi que le fait avec doigté, par exemple, E. Goffman notamment dans La mise en scène de la vie quotidienne. Dans des cas spécifiques et très limités, la métaphore théâtrale peut être isomorphe à la vie sociale et en conséquence elle peut aider à élaborer des opérations de spécifications de quelques utilités pour l'analyse.

La métaphore des jeux (7) fait des emprunts à la théorie du même nom, formulée en 1944 et depuis ayant connu diverses applications et généralisations. La théorie des jeux étudie la prise de décision en situation de concurrence, de conflit, de la part d'acteurs cherchant à satisfaire chacun ses propres intérêts. Ces derniers peuvent être convergents ou divergents selon le contexte. La théorie, afin de donner une formulation mathématique à des situations complexes et compliquées, doit procéder à des simplifications et postuler un principe de rationalité, dit principe du maximin, selon lequel un acteur tente, en évaluant les risques et les solutions avant d'arrêter le choix, de maximiser ses gains tout en sachant que son adversaire, doté de la même rationalité que lui, tentera de minimiser ses pertes.

L'utilisation de cette métaphore oblige le sociologue à présumer un type de comportements et des formes de conduites sociales gouvernées par un système de règles dont le déterminant majeur est le calcul des coûts et des bénéfices. Qu'il y ait des actions et des situations où les choix sont marqués par une rationalité basée sur la maximisation du profit, c'est évident, mais il s'agit de cas sporadiques, pour ne pas dire exceptionnels dans la vie quotidienne. L'action sociale est déterminée 
par les liens sociaux, par la conscience, l'intentionnalité, les réciprocités des perspectives, en somme par des logiques naturelles et des sentiments, le tout façonné et gouverné par les applications de modèles normatifs souvent affectés d'idiosyncrasie au calcul coût/bénéfice. Si le sociologue néglige cette donnée fondamentale, ou s'il prend en considération l'efficacité des moyens employés en faisant abstraction du profit recherché, l'usage de la métaphore ne produit qu'une explication incohérente et circulaire, tautologique. Norbert Elias l'a bien compris lorsqu'il a écrit dans Qu'est-ce que la sociologie?: "Les théories sociologiques qui présentent les choses de telle manière que les normes semblent être en quelque sorte les causes des relations sociales entre les hommes, ignorant qu'il pourra exister des relations humaines non normalisées et non réglementées, donnent des sociétés humaines une image aussi fausse que les théories qui ignorent le fait que les relations humaines, originellement non normalisées et non réglementées, puissent être soumises à la normalisation.» J'ajoute que Raymond Boudon a raison de répéter inlassablement que les apports de la théorie des jeux à l'analyse sociologique sont, jusqu'à ce jour, pratiquement inexistantes, qu'elle est inapplicable aux données observées, qu'elle doit être considérée comme une doctrine spéculative susceptible de rendre compte surtout de situations délibérément idéalisées. Sous cet angle-là, elle peut aider à formaliser quelques secteurs du langage des sciences sociales, à analyser certains mécanismes sociaux idéalisés, à faire de la simulation.

La métaphore du réseau (8), mise au point par Simmel, Moreno, RadcliffeBrown, Firth, Barnes, Bott, connaît un développement important depuis les années ' 70 , mais aussi des variations sémantiques à tel point divergentes qu'il est difficile de la penser de façon unitaire. En principe, elle postule que le réseau est l'ensemble des relations existantes entre l'ensemble des acteurs ou des éléments, relations ayant toutes les attributs de centralité, de cohésion et d'équivalence de positions. Or ces relations sont de nature disparate. Un réseau peut être organisé ou pas, ouvert ou fermé, défini ou indéfini. Pour le besoin de l'analyse, il faut en tracer les frontières et présumer qu'il constitue une totalité dont les éléments ont des degrés d'interdépendance. Dès lors sa structure ne peut être saisie qu'après coup et décrite avec des graphes. Elle ne préexiste pas aux relations, elle émerge des interactions.

Les atouts de cette métaphore sont évidents pour élaborer des hypothèses très riches (les structures émergent des relations et des interactions et puis elles contraignent les comportements), à condition toutefois que l'analyste présuppose que l'acteur agit de façon rationnelle, qu'il pense à son profit et qu'il fait des choix seulement après avoir comparé les avantages et les inconvénients. Nous rencontrons ici les mêmes difficultés que la théorie des choix et des anticipations rationnels a laissées en suspens. Pour les autres utilisations de cette métaphore, il suffit de renvoyer aux travaux de Bruno Latour et de Manuel Castells.

Il n'y a pas de critères pour comparer les métaphores usitées en sciences sociales, il n'y a pas de tests empiriques pour pondérer leur pertinence. L'analogie fonctionnelle et l'homologie sont rares car la similarité caractérisant des objets semblables est normalement indéfectible. L'isomorphisme de forme ou de structure entre deux systèmes à contenus différents n'est presque jamais réalisé. Et pourtant les métaphores nous permettent de «parler» de phénomènes qui se dérobent à une transcription directe; elles donnent de la vie à des nouveaux objets 
d'expérience, à des nouveaux champs perceptifs, voire à des nouveaux langages. Nous pouvons ainsi effectuer des opérations d'élaboration, de spécification et de semi-formalisation moyennant lesquelles la construction de modèles matériels, formels mathématiques, formels qualitatifs ou idéal-types, expérimentaux, simulés, est relativement faisable. Ces modèles, dérivés des métaphores mais transformées - quand cela est possible - en métaphores élaborées et déterminées, facilitent la réflexion théorique, l'évaluation des cohérences internes, l'adéquation avec l'objet, le dégagement de toutes les conséquences possibles.

Certes, les modèles sont irréels, ne permettent pas de relever des correspondances entre une mesure et la réalité sociale, néanmoins ils fournissent une aide puissante pour établir l'indispensable rapport logique entre les définitions nominales et les définitions opératoires. Artifices illustratifs, les modèles, bien qu'ils produisent des savoirs non cumulatifs, permettent de réduire les ambiguïtés et les équivoques du langage naturel, d'interpréter et d'expliquer les phénomènes sociaux, en bref de produire des effets d'intelligibilité.

Le langage des sciences sociales, et notamment de la sociologie, est agencé par une série de métaphores; elles commandent méthodologiquement la démarche comparative et rendent ainsi possible la connaissance par tâtonnement du monde social. Ce langage présente les deux structures argumentatives déjà décrites par Aristote: il va du fait à la règle, du fait au fait, du particulier au général (l'induction, l'exemple), et du général au particulier (la déduction et l'enthymème dont les prémisses probables peuvent rester implicites). Les métaphores de base établissent, implicitement ou explicitement, les prémisses communes, l'accord préalable sur les faits, les vérités, les présomptions, entre tous les membres d'une même communauté scientifique. Elles permettent de donner une assise aux arguments quasi logiques (arguments qui s'apparentent à l'identité et à la transitivité mais qu'on peut réfuter), à ceux s'appuyant sur l'expérience, à ceux fondant la structure du réel, à ceux procédant par dissociation de notion.

La conceptualisation métaphorique permet la fabrication de séries explicatives, de nourrir le raisonnement sociologique de nouvelles interprétations, de déployer la logique de l'argumentation au sens de Jean-Blaise Grize. Depuis des années Jean Claude Passeron nous rappelle que: «1. Les sciences empiriques sont des langages de description du monde... 2. [ Qu'] Il n'existe pas et il ne peut exister de 'langage protocolaire' unifié de la description empirique du monde historique. 3. [Que] La mise à l'épreuve empirique d'une proposition théorique ne peut jamais revêtir en sociologie la forme logique de la 'réfutation' ('falsification') au sens poppérien.»

Les métaphores jouent un rôle stratégique dans les sciences sociales, elles font «converger des preuves en forme logique différente dans un argumentaire d'ensemble, leurs arguments dans le langage de l'interprétation, leurs interprétations dans une théorie plausible», à condition toutefois, ajoute Passeron, qu'elles ne soient pas réduites, «à la répétition mécanique d'une ressemblance qui tourne en rond dans un modèle monotone d'interprétation, soustrait par la répétition à toute contre-interrogation comparative des différences.»

Dans son Traité de l'éloquence le cardinal Jacques Davy Du Perron disait la même chose mais avec des mots plus crus: «La métaphore est une petite similitude, il faut qu'elle passe vite, il ne faut pas s'y arrêter, quand elle est trop continue, elle est vicieuse et dégénère en énigme.» 
Les recherches en cours nous confirment dans l'idée que le rôle des métaphores en sociologie est important, qu'elles donnent aux méthodes de description et d'explication les formes de la logique argumentative, qu'au cœur des sciences historiques, pour conclure avec Max Weber, «sont inscrits en même temps le caractère provisoire de toutes les constructions idéal-typiques et la nécessité inéluctable d'en construire de toujours nouvelles » ${ }^{1}$.

Université de Lausanne

Ce texte a fait l'objet, le 21 juin 2002, de ma leçon d'adieu à l'Ecole doctorale «Education, Langage, Sociétés » de l'Université René Descartes-Paris 5 et au Cercle d'études Vilfredo Pareto de l'Université Paris-Sorbonne. Il est offert à Monsieur Jacques Coenen-Huther en signe d'estime et d'amitié. Ses travaux sociologiques, son talent d'enseignant, sa carrière académique exemplaire, sa droiture morale et intellectuelle, ont toujours suscité en moi gratitude et admiration.

Cette ébauche d'une recherche en cours sur les métaphores doit beaucoup à mes amis de toujours Jean-Blaise Grize, Jean-Claude Passeron et Pierre Livet, dont les derniers travaux ont été publiés dans «Enquête 2. L'argumentation, preuve et persuasion », Paris, EHESS, 2002. 